\title{
Amphiphilic poly-N-vinylpyrrolidone nanoparticles as carriers for non-steroidal anti-inflammatory drugs: Characterization and in vitro controlled release of indomethacin
}

\author{
A.N. KUSKOV ${ }^{1}$, A.A. VOSKRESENSKAYA ${ }^{1}$, A.V. GORYACHAYA ${ }^{1}$, M.I. SHTILMAN ${ }^{1}$, \\ D.A. SPANDIDOS ${ }^{2}$, A.K. RIZOS ${ }^{3}$ and A.M. TSATSAKIS ${ }^{4}$ \\ ${ }^{1}$ D.I. Mendeleyev University of Chemical Technology, 9 Miusskaya Square, Moscow 125047, Russia; \\ ${ }^{2}$ Department of Medicine, Laboratory of Virology, University of Crete; ${ }^{3}$ Department of Chemistry, \\ University of Crete, and FORTH - IESL, P.O. Box 2208; ${ }^{4}$ Department of Medicine, \\ Laboratory of Toxicology, University of Crete, Heraklion 71003, Crete, Greece
}

Received April 19, 2010; Accepted May 3, 2010

DOI: $10.3892 /$ ijmm_00000438

\begin{abstract}
Novel amphiphilic poly-N-vinylpyrrolidone derivatives with different molecular weight of hydrophilic PVP fragment and one secondary di-n-alkyl terminal hydrophobic fragment of different length were synthesized to compare their inclination for formation of nano-scaled micelle-like aggregates in aqueous media with previously studied primary n-alkyl terminated poly-N-vinylpyrrolidones. The behavior of amphiphilies in water solutions was studied and critical aggregation concentration values for prepared polymer samples were determined by fluorescence spectroscopy and compared with those for primary n-alkyl derivatives. Polymeric micelle-like particles with or without encapsulated drug were prepared using dialysis or solvent evaporation techniques. Indomethacin was incorporated into hydrophobic inner core of these nanoparticles as a typical model drug. Dynamic light-scattering studies determined that the average size of particles formed was from $90 \mathrm{~nm}$ up to $600 \mathrm{~nm}$ with monodisperse size distribution and the nanoparticle size slightly increased with the amount of indomethacin encapsulated into inner core of the particles. In vitro release experiments carried out at different medium $\mathrm{pH}$ values using indomethacin-loaded nanoparticles exhibited slow and steady drug release into the medium.
\end{abstract}

\section{Introduction}

In recent years, nano-scaled colloidal drug delivery systems such as liposomes, micelles and nanoparticles are extensively

Correspondence to: Dr Aristidis M. Tsatsakis, University of Crete, Department of Medicine, Laboratory of Toxicology, Heraklion 71003, Crete, Greece

E-mail: aris@med.uoc.gr

Key words: amphiphilic polymer, nanoparticle, micelle, drug delivery, controlled release, poly- $\mathrm{N}$-vinylpyrrolidone, indomethacin studied as one of the most promising strategies to achieve site-specific drug delivery (1-3). There have been vast efforts to use different natural or synthetic materials to develop efficient systems for targeted delivery of drugs $(4,5)$. Targeting the drug to the desired site would not only improve the therapeutic efficiency but also enable a reduction of the amount of drug which must be administered to achieve a therapeutic response, thus minimizing unwanted toxic effects.

Polymers have played a major role in the development of drug delivery systems. A wide variety of polymer particulate carriers has been devised for protecting biologically active molecules against inactivation by the organism and for controlling drug release in body fluids. The advantages of polymeric colloidal delivery systems are easy control of particle size, good structural stability and stability during long-term storage, solubilization of hydrophobic drugs and ability to deliver drugs showing low interactions with biocomponents such as proteins and cells $(6,7)$.

The numerous studies carried out showed that new technology for preparation of sub-micron nano-sized polymeric particles is required for providing new functions to drug delivery systems. The particles, usually $<1 \mu \mathrm{m}$, circulate in the blood stream without immobilization of capillaries, and permeate into the target cells through blood vessels (8). This particular dosage form is expected to help protect the incorporated drug from enzymatic attack in plasma by covering the incorporated drug in a hydrophobic core of the particles (9).

In most studies, block copolymers composed of hydrophilic and hydrophobic segments are used for development of drug delivery systems which can form a micellar structure $(1,10)$ with a hydrophobic compact inner core and a hydrophilic swollen outer shell in solvent, which is thermodynamically favorable for one block, but unfavorable for others $(11,12)$. Such copolymers usually consist of suitable biodegradable polymers such as poly(lactic/glycolic acid) (PLGA) (13), poly$\varepsilon$-caprolactone (PعCL) $(14,15)$, poly(L-lactide) (PLLA) (16) or polyethylene glycol (PEG) (17). Micelles made of these copolymers have been investigated with a novel type of 
sustained release system for targeting drugs to specific sites of the organism (18-20). However, in spite of the above mentioned advantages all listed polymers have significant disadvantages limiting their application in medical practice, such as low ability for additional polymer functionalization and non-proven safety of polymers and their derivatives after injection into the organism.

In addition, for any application of foreign material into the living organism, the material biocompatibility must be satisfied. For polymeric colloidal carriers for the parenteral and especially intravenous administration, the required host response must be a negative response to the carrier itself and to the drug-carrier conjugate. To achieve a long blood circulation half-life, we designed novel amphiphilic derivatives of poly-N-vinylpyrrolidone (PVP) as a basis for creation of highly-effective drug delivery systems. PVP has a long history of pharmaceutical applications and demonstrates a high degree of biocompatibility $(21,22)$.

The peculiarity of the prepared amphiphilic polymer structure is that these polymers consist of only one polymeric fragment (hydrophilic PVP) with one terminal long-chain aliphatic radical serving as hydrophobic fragment. Earlier we described an easy original two-stage method of synthesis of such polymers (23).

At concentrations above the so-called critical aggregation concentration (CAC), almost all amphiphilic polymer chains aggregate to form micelles, while below the CAC, only isolated chains are observed in the solution (12). Since most drugs have a hydrophobic character, these drugs can be easily incorporated into the particle core by a covalent or a non-covalent bonding through hydrophobic interactions with the experimental methods such as direct dilution, dialysis (5), salting out procedure, or solvent evaporation method (24). In our previous study (25) we demonstrated the possibility of immobilization of model proteins (Bowman-Birk soybean proteinase inhibitor and its derivatives) on nano-scaled aggregates made of amphiphilic PVPs.

The objective of the present study was to prepare a watercompatible dosage form of indomethacin on the basis of novel amphiphilic polymers. Indomethacin (IMC, Fig. 1a) has very low water solubility $\left(<0.03 \mathrm{~g} \cdot \mathrm{L}^{-1}\right)$ and is known as typical model drug for non-steroidal anti-inflammatory drugs (NSAIDs) $(26,27)$. It is widely used for treatment of rheumatoid arthritis, spondylitis and osteoarthritis (28). Thus, it is important to develop nano-scaled carrier system for the controlled release of indomethacin in order to achieve sustained medication clinically, especially for alleviating pain during sleep at night.

In our previous study, we reported on the preparation, characterization and in vitro controlled release study of indomethacin-loaded polymeric nano-scaled particles composed of hydrophobically primary n-alkyl $\alpha$-end-capped poly-N-vinylpyrrolidone (Fig. 1b) (29). This is a continuing study on micelle-like nanoparticles prepared using PVP amphiphilic derivatives.

As the major role of drug loading process is played by hydrophobic interactions between poor-soluble drug and nanoparticle hydrophobic inner core, in this study we prepared new secondary di-n-alkyl terminated poly-N-vinylpyrrolidones (Fig. 1c) to obtain nanoparticles with more hydrophobized inner core in order to produce more stable drug delivery system with greater loading efficiency comparing to monon-alkyl $\alpha$-end-capped PVPs.

To evaluate capability of carriers for drugs, we prepared IMC-loaded polymeric particles by different methods. The characteristics of these particles were investigated through dynamic light-scattering (DLS), transmission electron microscopy (TEM) and fluorescence probe technique. In addition, the drug loading efficiency of incorporated IMC was investigated by ultraviolet (UV) spectrophotometer. The drug release properties of prepared nanoparticles were also investigated.

\section{Materials and methods}

Materials. N-vinylpyrrolidone (VP) and indomethacin (IMC) were obtained from Sigma (USA). Substrate for electron microscopy $-0.2 \%$ polyvinylformal, was from Merk (Germany). All other chemicals used were reagent grade and used as purchased without further purification. All solvents and components of buffer solutions were analytical grade preparations. Distilled-deionized water was prepared with a Milli-Q Plus System (Millipore, USA).

Synthesis of $N$-vinylpyrrolidone amphiphilic polymer. $\mathrm{N}$-alkyl terminated (Amph-PVP) and di-n-alkyl terminated $\left(\mathrm{Amph}_{2}{ }^{-}\right.$ PVP) amphiphilic $\mathrm{N}$-vinylpyrrolidone polymers were prepared using originally developed two-stage method as described in our previous studies $(23,25)$. In the first stage, poly-Nvinylpyrrolidone (PVP) with one terminal carboxylic group was synthesized by free-radical polymerization of N-vinylpyrrolidone in the presence of initiator (azobisisobutyronitrile (AIBN) and chain-transmitter [mercaptoacetic acid (MAA)]. The reaction was carried under dry argon atmosphere in dioxane solution for $2.5 \mathrm{~h}$ at $60^{\circ} \mathrm{C}$. The yield of polymers was $75 \% \div 90 \%$. MW values were determined by titration or steam osmometry using a Knauer osmometer (Germany). Polydispersity of one end functionalized polymers was determined by high-performance liquid chromatography (GFHPLC; TSK Gel G4000PwxL; Toso Co., Ltd., Japan). For PVP 2000, PVP 3500, PVP 5000 and PVP 9000 samples Mw/Mn values were $1.10,1.12,1.12$ and 1.15 , respectively.

On the second stage, hydrophobic n-alkyl or di-n-alkyl groups (octadecyl, di-n-octadecyl, di-n-dodecyl, di-n-hexyl) were attached to reactive terminus of the PVP molecule. For this purpose, the solution of carboxy-PVP in isopropanol was supplemented with an excess of $\mathrm{N}, \mathrm{N}$-dicyclohexylcarbodiimide (DCC) in an equal volume of the same solvent. The mixture was stirred at $5^{\circ} \mathrm{C}$ for $2 \mathrm{~h}$, and then excess of appropriate aliphatic amine dissolved in isopropanol was added. The mixture was incubated for $3 \mathrm{~h}$ at $65^{\circ} \mathrm{C}$ until the full connection of hydrophobic alkyl groups. Then, polymers were isolated by precipitation, dried to constant weight and their yield was determined.

Preparation of indomethacin-loaded nanoparticles from amphiphilic poly-N-vinylpyrrolidone. Amphiphilic N-vinylpyrrolidone polymeric nanoparticles were prepared using dialysis and solvent evaporation method. Indomethacin (IMC) was used as model drug with hydrophobic nature. 
<smiles>COc1ccc2c(c1)c(CC(=O)O)cn2C(=O)c1ccc(Cl)cc1</smiles>

a) Indomethacin

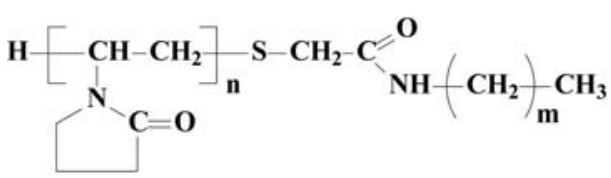

b) Amph-PVP

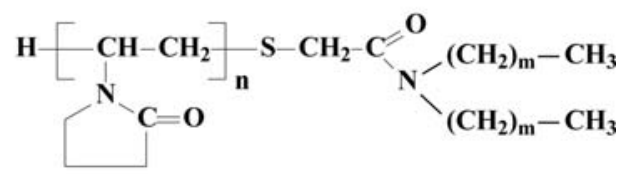

c) $\mathrm{Amph}_{2}-\mathrm{PVP}$

Figure 1. Structural formula of indomethacin (a), n-alkyl terminated Nvinylpyrrolidone amphiphilic polymer (b) and di-n-alkyl terminated amphiphilic polymer (c).

In dialysis method amphiphilic PVP was dissolved in dimethylformamide followed by the addition of IMC with various weight ratios to polymer $(1: 0.1 \div 1: 1)$ and stirred at room temperature. To form IMC-loaded nanoparticles and remove free IMC, the solution was dialyzed for $24 \mathrm{~h}$ against 3 liters of ultra pure water using regenerated cellulose dialysis membranes (molecular weight cut-off: $3.5 \times 10^{3}$ and $6 \times 10^{3} \div$ $8 \times 10^{3}$, Membrane Filtration Products Inc., USA). The nanoaggregates solution was sonicated using ultrasonic dispergator Sonoplus HD 2070 (Bandelin, Germany), and then centrifuged (Heraeus, Martin Christ GmbH, Germany) to remove unloaded IMC and aggregated particles.

In the solvent evaporation (emulsion) method different weight ratios of amphiphilic PVP and IMC were dissolved in small amount of chloroform. This mixture was then emulsified in an aqueous phase with ultrasonic dispergator (Sonoplus HD 2070, Bandelin). The organic solvent was then removed and the resulting suspension concentrated by evaporation under reduced pressure (rotary evaporator Laborota 4010, Heidolph, Germany).

IMC-loaded nanoparticle suspensions, obtained in both processes, were frozen and lyophilized by Alpha I-4LD freeze dryer system (Martin Christ GmbH, Germany) to obtain dried nanoparticle products. Thermogravimetric analysis of freeze-dried nanoparticles confirmed that there were no residues of organic solvents left in the drug-loaded particles. The plain nanoparticles without IMC were prepared by the same methods, but without addition of indomethacin.

Particle characterization. To estimate the critical aggregation concentration (CAC) values for amphiphilic PVP samples, fluorescence measurement was carried out using pyrene as fluorescent probe. The method is based on solubilization of hydrophobic pyren by polymeric nanoparticles. For this purpose, aliquots of $10 \mu \mathrm{l}$ of pyrene solution in acetone $(10 \mathrm{mg} /$ $\mathrm{ml})$ per test tube were dried under vacuum. The tubes were supplemented with $2 \mathrm{ml}$ of serial dilutions $\left(10^{-4} \div 10^{-10} \mathrm{M}\right)$ of various PVP samples, and shaken overnight at room temperature. The samples were filtered through $0.2 \mu \mathrm{m}$ filter to remove the non-solubilized pyrene, and the fluorescence intensity of solubilized pyrene was measured using a Hitachi 650-10 S spectrofluorophotometer (Hitachi Instruments Inc., Japan). The emission wavelength was $390 \mathrm{~nm}$ for excitation spectra.

Average size and size distribution of nano-aggregates were determined by dynamic light scattering (DLS) using N5 Submicron particle size analyzer (Beckman Coulter Inc., USA). Dynamic light scattering experiments were conducted by means of a light scattering spectrometer with vertically polarized incident light of wavelength $\lambda=532 \mathrm{~nm}$ supplied by a diode laser. The laser beam was linearly polarized in the direction perpendicularly to the scattering plane. In the present study, the full homodyne intensity autocorrelation function was measured at different scattering angles in the range $30-150^{\circ}$ with an ALV-5000 multiple- $\tau$ digital correlator that covered a dynamic range of about ten decades. The correlation functions were recorded in the real-time 'multiple- $\tau$ ' mode of the correlator, in which 256 time channels are logarithmically spaced over an interval ranging from $0.2 \mu \mathrm{sec}$ to almost an hour. For each sample, the mean diameter of six determinations was calculated by applying multimodal analysis.

For morphological examinations, nanoparticles were analyzed with transmission electron microscopy (TEM) using apparatus JEOL JEM-2100 (Germany) at a voltage of $120 \mathrm{kV}$. For sample preparation, a drop of particle suspension was placed on substrate from $0.2 \%$ solution of polyvinylformal applied on copper mesh.

Drug loading characterization. The amount of IMC loaded into inner core of Amph-PVP and $\mathrm{Amph}_{2}$-PVP particles was investigated using UV spectrophotometer (Hitachi 650-10 S). To remove unbound and immobilized IMC on particle surface, the solution was sonicated, centrifuged and then lyophilized. The precipitate containing unloaded IMC was dissolved in $50 \%$ ethanol solution and IMC amount in it was determined by measuring UV absorbance at $318 \mathrm{~nm}$. The standard solutions were prepared at concentrations ranging from 0.01 to $0.10 \mathrm{~g} \cdot \mathrm{L}^{-1}$. Drug loading capacity (DLC) and drug loading efficiency (DLE) were calculated using equation (i) and (ii), respectively.

$$
\begin{aligned}
& \operatorname{DLC}(\%)=\frac{A-B}{C} \times 100 \\
& D L C(\%)=\frac{A-B}{A} \times 100
\end{aligned}
$$

where $A$ is the total weight of IMC used, $B$ is the weight of unloaded IMC in the precipitate after centrifugation and $C$ is the weight of amphiphilic polymer used. In order to study the effect of $\mathrm{pH}$ on IMC loading and capacity, the PBS solutions with $\mathrm{pH}$ values $6.1,6.7,7.4$ and 8.0 were used to prepare IMC-loaded nanoparticle solutions. 
Table I. Synthesized amphiphilic N-vinylpyrrolidone polymers and their properties.

\begin{tabular}{|c|c|c|c|c|c|c|}
\hline \multirow[b]{2}{*}{ Sample } & \multicolumn{2}{|c|}{$\begin{array}{l}\text { Hydrophilic fragment } \\
\text { number average } \\
\text { molecular weight }\end{array}$} & \multirow[t]{2}{*}{$\begin{array}{l}\text { Hydrophobic } \\
\text { fragment } \\
\text { type }\end{array}$} & \multirow[b]{2}{*}{$\mathrm{CAC}, \mu \mathrm{M}$} & \multicolumn{2}{|c|}{$\begin{array}{l}\text { Particle size } \\
\text { mean } \pm \mathrm{SD} \\
(\mathrm{nm})\end{array}$} \\
\hline & Titration $^{\mathrm{a}}$ & Osmometry $^{\mathrm{b}}$ & & & Dialysis $^{\mathrm{c}}$ & Emulsion $^{\mathrm{d}}$ \\
\hline PVP-DD 3500 & 3510 & 3518 & di-n-dodecyl & 7.4 & $187 \pm 9$ & $151 \pm 6$ \\
\hline PVP-DD 25000 & 5050 & 5021 & di-n-dodecyl & 8.4 & $204 \pm 7$ & $162 \pm 3$ \\
\hline PVP-DD 29000 & 9030 & 9012 & di-n-dodecyl & 9.8 & $237 \pm 2$ & $208 \pm 5$ \\
\hline PVP-Hex 25000 & 5050 & 5021 & di-n-hexyl & 15.6 & $342 \pm 8$ & $325 \pm 1$ \\
\hline PVP-OD 25000 & 5050 & 5021 & di-n-octadecyl & 4.3 & $292 \pm 2$ & $277 \pm 6$ \\
\hline PVP-OD 5000 & 5050 & 5021 & n-octadecyl & 8.9 & $131 \pm 8$ & $104 \pm 7$ \\
\hline
\end{tabular}

${ }^{a}$ Determined by potentiometric titration of end carboxyl groups. ${ }^{b}$ Determined by steam osmometry method. ${ }^{c}$ Estimated for Amph-PVP and Amph-PVP ${ }_{2}$ nanoparticles prepared by dialysis method. ${ }^{\mathrm{d} E s t i m a t e d ~ f o r ~ A m p h-P V P ~ a n d ~ A m p h-P V P ~}{ }_{2}$ nanoparticles prepared by solvent evaporation technique.

In vitro IMC release measurements. Release studies were conducted at $37^{\circ} \mathrm{C}$ under magnetic stirring. PBS buffer $(10 \mathrm{ml}$ of 0.1 M) (pH 7.4) IMC-loaded Amph-PVP and $\mathrm{Amph}_{2}$-PVP nanoparticles of different composition were enclosed in a dialysis bag (molecular weight cutoff: 1000), which was placed in $500 \mathrm{ml}$ of PBS buffer at $\mathrm{pH}$ 7.4. At predetermined time intervals $1.0 \mathrm{ml}$ of buffer solution outside the dialysis bag was removed and replaced with $1.0 \mathrm{ml}$ of fresh buffer solution. The amount of released IMC was analyzed by UV spectrophotometry at $318 \mathrm{~nm}$. The standard solutions were prepared with PBS ( $\mathrm{pH} 7.4$ ) at concentrations ranging from $1.0 \times 10^{-3}$ to $1.0 \times 10^{-1} \mathrm{~g} \cdot \mathrm{L}^{-1}$.

\section{Results and Discussion}

Synthesis of $N$-vinylpyrrolidone amphiphilic polymers. For preparation of poly-N-vinylpyrrolidone amphiphilic derivatives with one terminal n-alkyl or di-n-alkyl hydrophobic fragment we used the original two-stage process. First we intended to prepare VP homopolymer $\alpha$-end-capped with functional carboxylic group by free radical polymerization of monomer in the presence of AIBN as initiator and MAA acid as chain growth regulator and carboxyl group source. Such a scheme of polymerization allowed for preparation of semitelechelic PVP with one end carboxyl group, controllable molecular weight and low polydispersity $(\mathrm{Mw} / \mathrm{Mn} \sim 1.1)$. The structure of obtained polymers was confirmed by FT-IR spectral analysis, ${ }^{1} \mathrm{H}-\mathrm{NMR}$ spectroscopy and by agreement of molecular weight values determined by potentiometric titration of PVP carboxyl groups (on the basis of one carboxyl group per one polymer molecule) and by osmometry. The results of the investigation of this process and its kinetics are of single interest and will be reported in a separate study shortly.

In the second stage, prepared semitelechelic PVP was treated by appropriate amount of long-chain aliphatic amine (octadecyl, di-n-octadecyl, di-n-dodecyl or di-n-hexyl amine) in the presence of DCC in order to attach single hydrophobic fragment to terminal carboxylic group of each PVP molecule. The total substitution of carboxyl groups was confirmed by
FT-IR and NMR analysis and by titration under the same conditions as for semitelechelic polymer molecular weight estimation.

Applied two-staged method allows preparation of amphiphilic PVP derivatives with different size of hydrophilic and hydrophobic fragments (Table I) which can be easily controlled for achieving optimal polymer properties. As prepared polymers are planned to be used as drug carriers, the biocompatibility of the whole system is of vital importance. That is why it was decided to limit higher molecular weight of PVP fragment by 10000 as it is known that low-molecular PVPs possess high biocompatibility (PVP with molecular weight $8000-1000$ is widely used as component of blood substitutes).

The main object of our investigation was to evaluate perspective of more hydrophobized di-n-alkyl tetminated amphiphilic PVPs as a basis for the drug delivery system, so we compared the yields of prepared $\mathrm{Amph}_{2}$-PVP and Amph-PVP. The total yield of $\mathrm{Amph}_{2}$-PVP (70-80\%) was lower than the yield of most Amph-PVPs (85-95\%). Obviously, it can be explained by lower rates of coupling reaction between PVP carboxylic group and secondary amines in regard to primary amines. Also the low yields were obtained for amphiphilies with the lowest PVP fragment molecular weight $\mathrm{Mn}=2000$ (yield: 60-70\%). Such low polymer yield for these samples can be explained by large fraction of low-molecular products in reaction mixture which cannot be isolated by methods used for amphiphilic polymer preparation. Finally, the low yields were determined for amphiphilic polymers with terminal di-n-octadecyl group (yield: $55-65 \%$ ) due to its very high hydrophobicity and sterical volume. That is why, amphiphilic polymers with low molecular weight of hydrophilic PVP fragment and with di-n-octadecyl hydrophobic group cannot be observed as a promising basis for indomethacin delivery systems from a technological and practical point of view.

For the above-mentioned reasons, for the following investigations, we chose two series of amphiphilic polymers (Table I) - polymers with PVP fragment molecular weight 


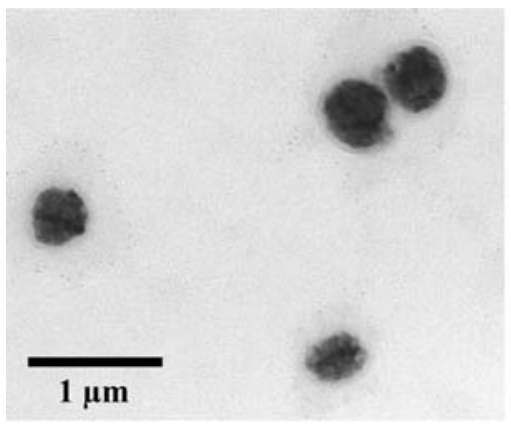

(a)

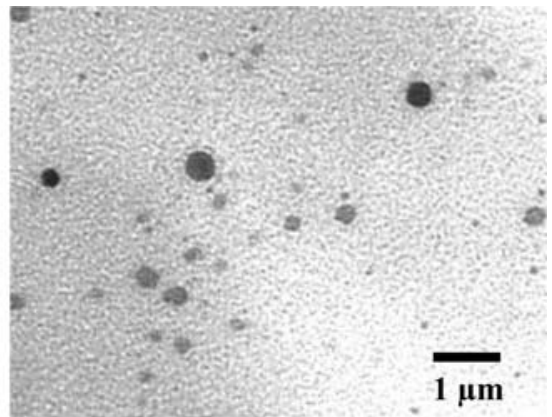

(b)

Figure 2. Transmission electron micrographs: (a) nanoparticles prepared from PVP-DD 2 5000; (b) $\mathrm{PVP}^{-\mathrm{DD}_{2}} 5000$ nanoparticles loaded with IMC (weight ratio $1: 1)$.

5000 and different di-n-alkyl hydrophobic fragment (di-noctadecyl, di-n-dodecyl, di-n-hexyl) and polymers with di-ndodecyl hydrophobic terminal group and different molecular PVP fragment weight $(3500,5000,9000)$. For comparison with primary n-alkyl hydrophobized PVP, we synthesized and studied polymers with molecular weight of PVP fragment 5000 and n-octadecyl hydrophobic end group.

Amph $_{2}$ PVP nanoparticle formation. As prepared PVP amphiphilic derivatives contain hydrophilic and hydrophobic fragments, at certain concentrations in aqueous media greater than some critical concentration (so-called critical aggregation concentration) they can aggregate with formation of core-shell type polymeric nanoparticle structures. Due to the hydrophobic character of terminal long-chain fragments, di-n-alkyl domain will be oriented towards the core of the polymeric nanoparticles while hydrophilic PVP is oriented in an outward direction as an outer shell of the polymeric nanoparticles.

To obtain information on critical aggregation concentration (CAC) for different $\mathrm{Amph}_{2}$-PVP samples, to compare it with those of Amph-PVP and to study the influence of polymer hydrophilic/hydrophobic fragment size and type on the process of nanoaggregates formation, we estimated the fluorescence excitation spectra of pyrene at various concentrations of amphiphilic polymers. Excitation wavelength was $390 \mathrm{~nm}$ and pyrene concentration was kept constant at $6.0 \times 10^{-6} \mathrm{M}$. Pyrene was chosen as fluorescent probe because of its photochemical properties and remarkably long life-time suitable for an effective probe (30). Hydrophobic and very low-soluble in water pyrene preferentially solubilizes into the interior of the hydrophobic fragments of amphiphilic PVPs, so that it moves from water environments to hydrophobic particle cores. Therefore, the fluorescence intensity is affected by the change in the polymer concentration.

CAC values for amphiphilic polymer samples with different size of hydrophilic and hydrophobic parts are presented in Table I. CAC values for most polymer samples are in micromolar range, which is much lower than that of common low-molecular weight surfactants. This result indicates that $\mathrm{Amph}_{2}-\mathrm{PVP}$ similarly to Amph-PVP systems can retain a micelle-like structure even in greatly diluted solutions, featuring stable polymeric particles which may be useful as drug vehicles.

As expected the CAC of di-n-alkyl PVP derivatives was lower than of n-alkyl amphiphilies due to higher hydrophobicity. Also, CAC values slightly decreased with decreasing hydrophilic PVP fragment molecular weight and dramatically decreased with increasing hydrophobic anchor chain length. Obtained data fully corresponds with results of other investigators $(31,32)$, reporting that the micellization process is determined mainly by the nature and the length of the hydrophobic block, where the nature of soluble hydrophilic block has only a slight effect on the onset of micelle formation.

The lowest CAC value among PVP di-n-alkyl derivatives was obtained for PVP-OD 25000 , so it possesses the highest inclination for self-assembling. However, the application of this particular polymer is limited by its rather low yield on synthesis stage and low solubility in water at concentrations higher than CAC, similarly to all other di-n-octadecyl derivatives. Therefore, we chose the PVP-DD 5000 polymer as the main object for further investigations as it can be prepared with high yield and its ability to form stable and compact micelle-like nano-scaled particles is commensurable with those of PVP-OD 5000, which is one of the most perspective polymers with terminal n-alkyl hydrophobic group prepared and investigated earlier.

Characterization of $\mathrm{Amph}_{2}-\mathrm{PVP}$ nanoparticles. The morphology of self-assembled aggregates formed spontaneously by different amphiphilic polymers in aqueous media is quite diverse. Generally, it is supposed that amphiphilic polymers containing hydrophilic and hydrophobic blocks at concentrations higher than their CAC value produce particles close to spherical form. In the present study, we studied $\mathrm{Amph}_{2}-\mathrm{PVP}$ polymeric nanoparticles prepared both by dialysis and solvent evaporation methods using transmission electron microscopy. The obtained microphotographs confirmed formation of nano-sized particles with spherical shape (Fig. 2). The size and size distribution of nanoparticles prepared by dialysis or solvent evaporation were measured by means of a dynamic light scattering method (DLS).

Fig. 3 demonstrates experimental correlation functions at different scattering angles accompanied by the distribution 

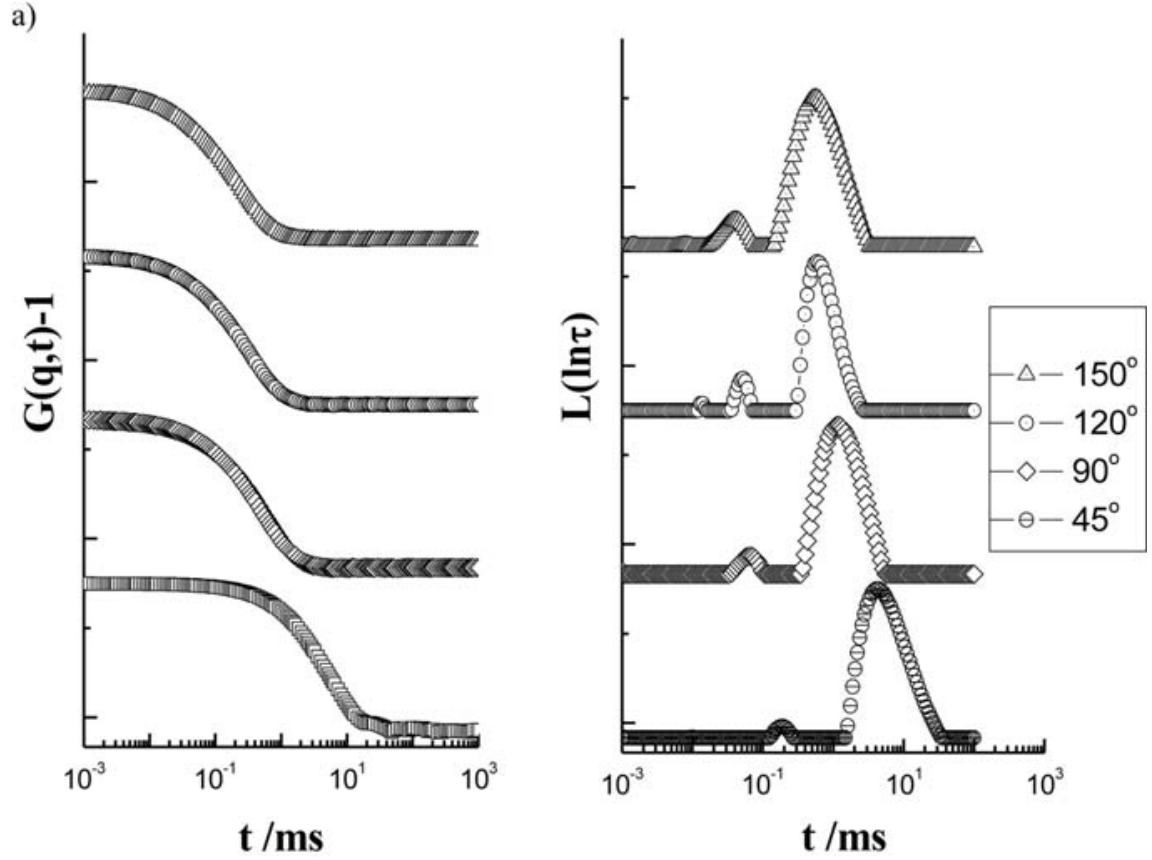

b)

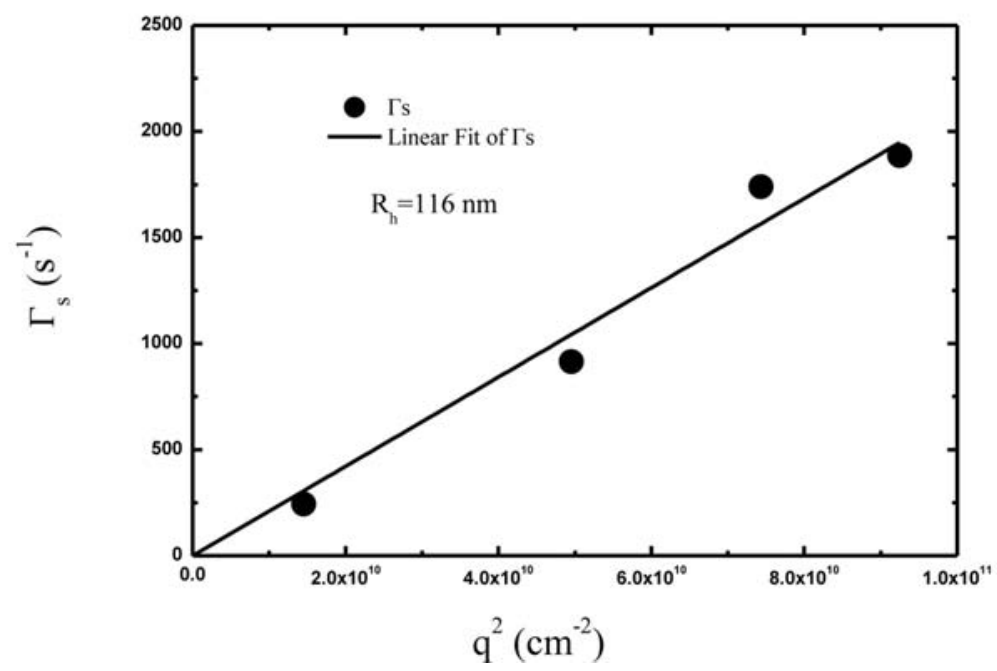

Figure 3. a) Experimental correlation functions at different scattering angles accompanied by the distributions of relaxation times of the experimental correlation functions for PVP-OD 5000 amphiphilic polymer. b) Estimation of hydrodynamic radius. The light scattering process defines a wave vector $\mathrm{q}=(4 \pi \mathrm{n} / \lambda) \sin (\theta / 2)$, where $\lambda \mathrm{s}$ the wavelength of the incident light in vacuum, $\theta$ is the scattering angle, $\mathrm{n}$ is the refractive index of the medium and $\Gamma_{\mathrm{s}}$ is the characteristic relaxation rate $\left(\Gamma_{\mathrm{s}}=1 / \tau_{\mathrm{s}}\right)$.

of relaxation times of the experimental correlation functions on the example of PVP-OD 5000 amphiphilic polymer with a hydrodynamic radius of $116 \pm 5 \mathrm{~nm}$.

Based on the DLS measurements (Table I), the average diameter of prepared particles was from $150 \mathrm{~nm}$ up to $350 \mathrm{~nm}$ which is much higher than for mono-alkyl terminated amphiphilies (30-200 nm). Fig. 4a shows the typical size distribution of $\mathrm{Amph}_{2}-\mathrm{PVP}$ nanoparticles. The size distribution of examined $\mathrm{Amph}_{2}$-PVP samples showed a narrow and monodisperse unimodal pattern with slightly wider distribution comparing to Amph-PVP as shown in Fig. 4b. The size of $\mathrm{Amph}_{2}-\mathrm{PVP}$ nanoparticles increased with the molecular weight of hydrophilic polymeric fragment and with decreasing of hydrophobic terminal group length. These results fully correspond with data obtained for CAC studies.

The influence of polymer concentration on the size of prepared particles was also studied (Table II). At low concentrations near CAC values, $\mathrm{Amph}_{2}-\mathrm{PVP}$ formed micelle-like spherical particles in water solutions with the average size $<300 \mathrm{~nm}$. At higher polymer concentrations (dozen times higher than $\mathrm{CAC}$ ), polymeric nanoparticles associated with the formation of larger aggregates (up to $1.5 \mu \mathrm{m}$ ) with complex structure. Also, the increased hydrophobic nature of di-n-alkyl $\alpha$-end-caped PVP derivatives provided poor solubility in water, and that is why we failed to obtain stable suspension of nanoparticles for $\mathrm{Amph}_{2}$-PVPs with molecular 


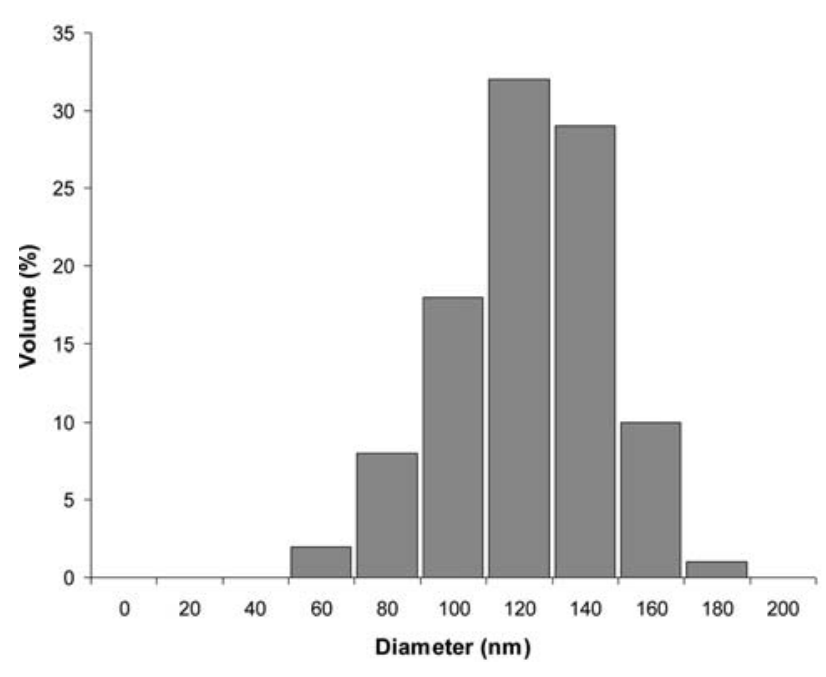

(a)

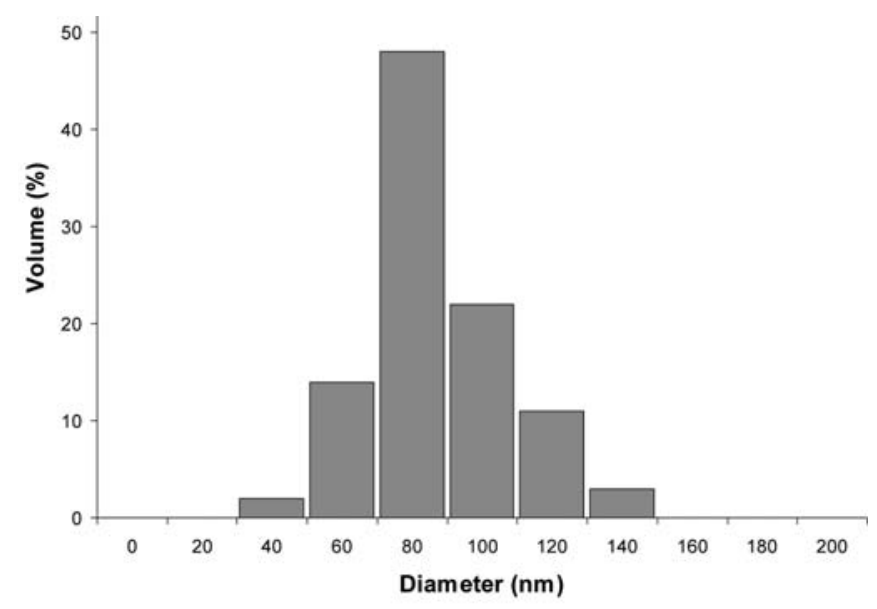

(b)

Figure 4. Typical size distribution profile of $\mathrm{Amph}_{2}$-PVP nanoparticles (PVP-DD 2 5000) (a) and Amph-PVP nanoparticles (PVP-OD 5000) (b) by dynamic light scattering measurement.

weight 3500 . In this case, at concentrations even a little higher than CAC, prepared solutions contained very large aggregates $(>1 \mu \mathrm{m})$ or were very turbid with formation of sediment fracture and unsuitable for DLS investigations. The size of other $\mathrm{Amph}_{2}$-PVPs particles at a high polymer concentration can be in a manner controlled by sonication or particle preparation conditions, but not so easily as for n-alkyl hydrophobized PVP which is further evidence of stronger hydrophobic interactions between di-n-alkyl groups.

For all $\mathrm{Amph}_{2}$-PVP samples the size of the particles prepared by emulsion method was smaller than of those prepared by dialysis (Table II). Thus, the solvent evaporation method is more suitable and preferred for preparation of polymeric nanoparticles.

To examine the existence of any residual organic solvents, nanoparticle solution obtained through dialysis or solution evaporation procedure was investigated by gas chromatography mass spectroscopy (GCMS QP-2010, Shimadzu, Japan). In the spectrum, the peaks of solvents used were not detected.

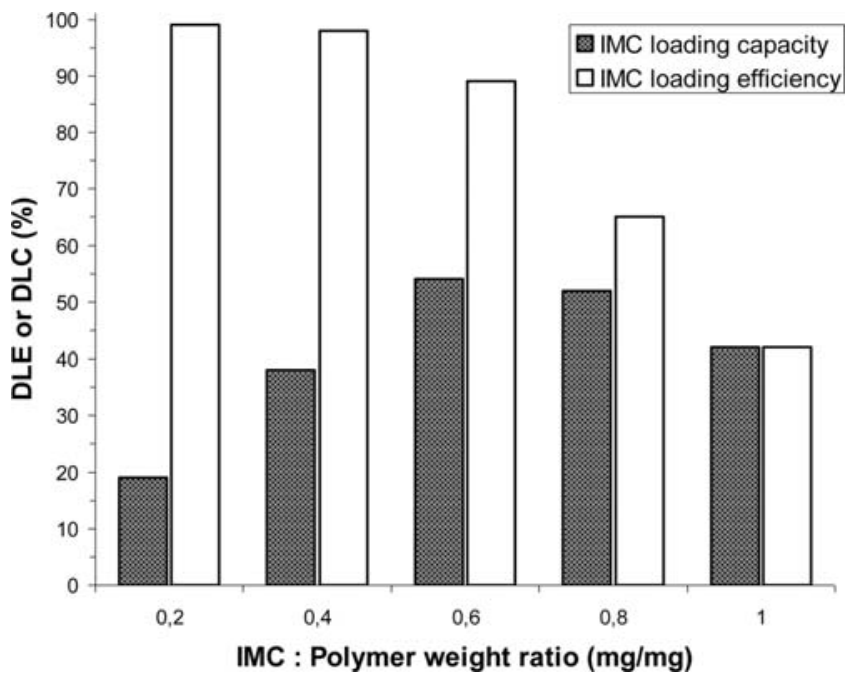

Figure 5. Influence of total amount of used IMC on drug loading efficiency and drug loading capacity of PVP-DD 25000 nanoparticles.

Thus, it indicated that organic solvent used for preparation of nanoparticles was completely removed through the dialysis or rotary evaporation process.

Drug loading studies. Indomethacin was dissolved in a small amount of the solvent, added dropwise to polymer solution and then IMC-loaded nanoparticles were prepared using the same techniques (dialysis or solvent evaporation) as for hollow nanoparticles. After removing of the solvent the IMC molecules were gradually entrapped into the hydrophobic microdomains of $\mathrm{Amph}_{2}$-PVP aggregates via self-assembly.

The introduction of IMC into Amph-PVP nanoparticles influences particle size. The results obtained by DLS measurements of IMC-loaded $\mathrm{Amph}_{2}$-PVP nanoparticles showed that at a low polymer concentration (near CAC value) their mean sizes were in the range of 200-400 nm depending on amount of introduced IMC, which is larger than size of the blank $\mathrm{Amph}_{2}$-PVP aggregates (Table II). This result fully corresponds with other studies $(33,34)$ where the increase of the size of IMC-loaded micelles after the loading capacity exceeded $25 \%$ comparing with blank micelles.

In the case of higher polymer concentration (dozen times higher CAC value) introduction of IMC leads to a large decrease of loaded nanoparticle size close to the size values obtained for low $\mathrm{Amph}_{2}$-PVP concentration (Table II). We can assume that in this case the introduction of hydrophobic IMC molecules in the system compacts and regulates the structure of colloid aggregates. At low ratio of IMC to AmphPVP in the mixture the strength of interaction between polymer chains prevails, but by increasing the hydrophobic drug concentration in the mixture the main contribution is made by hydrophobic interactions between IMC and the amphiphilic polymer. As a result, the small micelle-like IMC-loaded nanoparticles are formed instead of large complex polymeric aggregates.

Fig. 5 demonstrates the influence of totally used IMC amounts on the drug loading of several PVP-DD 2000 nanoaggregates. It is shown that, IMC loading capacity increases with an increase of added IMC and reached a maximum value 


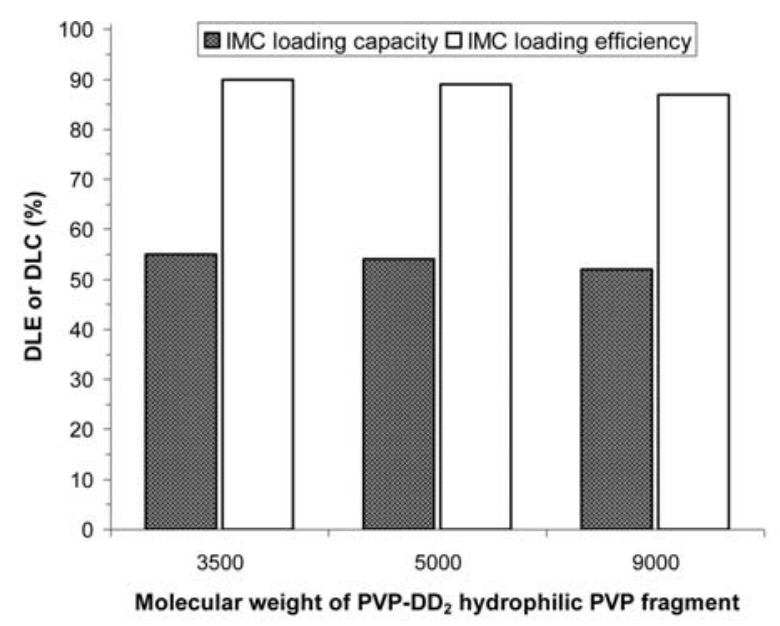

(a)

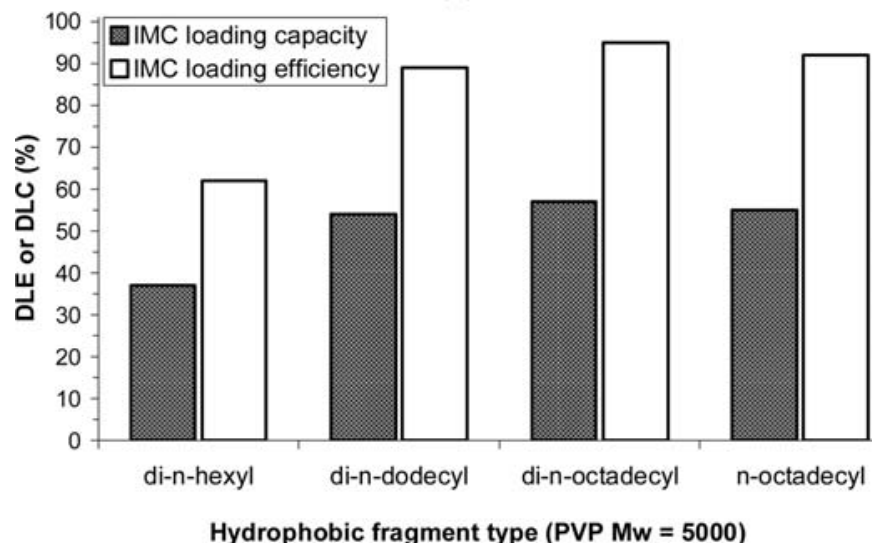

(b)

Figure 6. Effect of the amphiphilic PVP derivative hydrophilic (a) and hydrophobic (b) fragment structures on IMC loading efficiency and IMC loading capacity (IMC/polymer weight ratio: $0.6 \mathrm{mg} / \mathrm{mg}$ ).

of $\sim 50 \%$, with following decrease to $\sim 40 \%$. On the other hand, the drug loading efficiency reached $\sim 100 \%$ when the loading capacity was rather low $(<40 \%)$, indicating that IMC used was completely encapsulated inside the polymeric nanoparticle inner cores. This agreed with experimental observations, as the prepared colloid solutions in this case were stable with no noticeable precipitate. The further increasing of the IMC amount led to the gradual appearance of precipitate in the system. As a result, the drug loading efficiency decreased.

Moreover, the DLS measurements showed that, similar to Amph-PVP polymers, in case of $\mathrm{Amph}_{2}$-PVPs increasing IMC loading capacity $>50 \%$ leads to abrupt increase of polymeric particle size caused by aggregation between particles and formation of more complex structures due to the hydrophobic interactions. Therefore, in the drug loading experiments, the weight ratio of polymer to IMC was fixed to less than 1:1 for all samples. Drug loading efficiency for $\mathrm{Amph}_{2}$-PVP particles prepared by emulsion method is in most cases higher than that for particles prepared by dialysis.

The influence of the di-n-alkyl PVP derivative structure on IMC loading capacity and loading efficiency of IMC was also studied. Fig. 6a shows that molecular weight of PVP hydrophilic fragment showed only a slight change of IMC

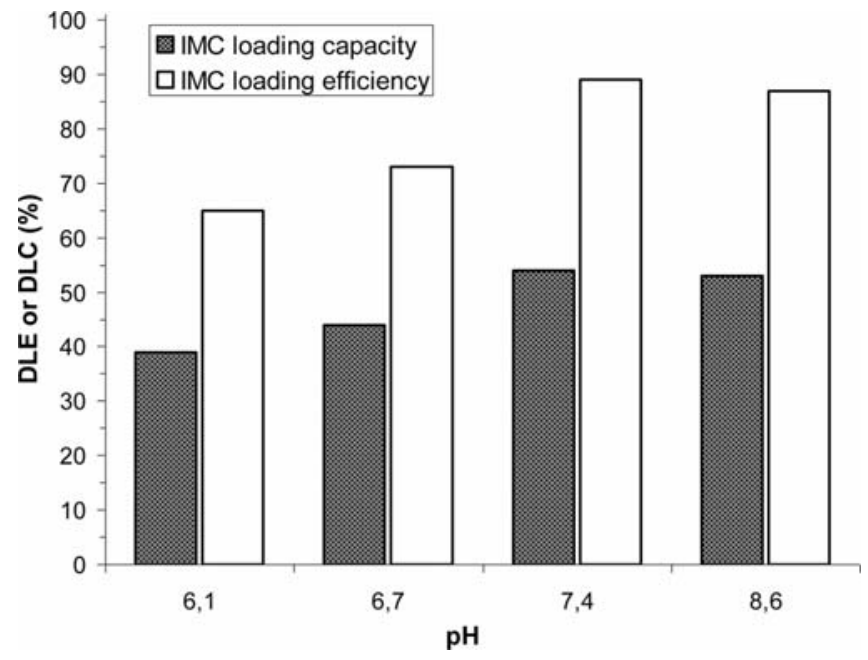

Figure 7. Effect of media $\mathrm{pH}$ on IMC loading efficiency and loading capacity of PVP-DD 5000 nanoparticles (IMC/polymer weight ratio: 0.6 mg/mg).

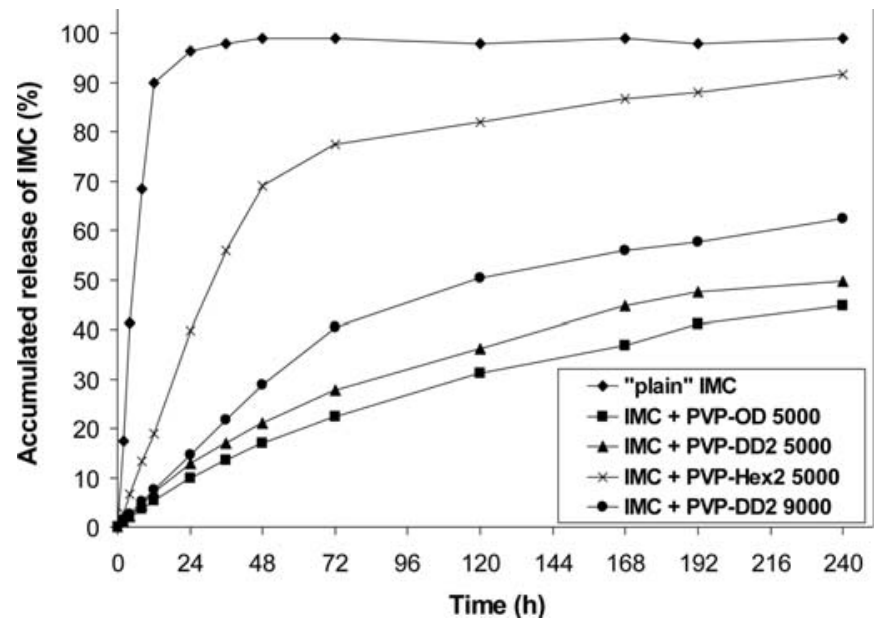

Figure 8. In vitro release profiles of IMC from different $\mathrm{Amph}_{2}-\mathrm{PVP}$ and Amph-PVP nanoparticles in $\mathrm{pH}$ 7.4 $\mathrm{PBS}$ at $37^{\circ} \mathrm{C}$.

loading capacity and loading efficiency. On the other hand, the increase of di-n-alkyl anchor length noticeably increased IMC loading efficiency (Fig. 6b). The best results were obtained for PVP-OD 5000 (for this polymer sample we succeeded in preparing IMC-loaded nanoparticles only in a few experiments and at low concentrations due to its hydrophobic nature) and PVP-DD 5000 and are not inferior to those for $n$-alkyl terminated PVP-OD 5000. The obtained data fully correspond with CAC values and self-assembly behavior of $\mathrm{Amph}_{2}$-PVPs studied by fluorescence analysis. Such polymers have lowest CAC values and can form nanoparticles and solubilize IMC more easily due to their optimal hydrophobic/hydrophilic balance. For PVP-DD 5000 polymer we can prepare nanoparticles with relatively high DLE of $41.4 \%$ at loading capacity of $50 \%$.

Fig. 7 shows the effect of PBS buffer solution $\mathrm{pH}$ values on the DLE and DLC of polymeric nanoparticles. Both DLE and DLC were lower at $\mathrm{pH} 6.1$ and 6.7 than at 7.4 and 8.8. 
Table II. Particle size parameters of IMC-loaded Amph-PVP and $\mathrm{Amph}_{2}-\mathrm{PVP}$ nanoparticles $\left(\mathrm{C}_{\mathrm{p}}-\right.$ amphiphilic polymer concentration).

Particle size mean \pm SD $(\mathrm{nm})$

\begin{tabular}{|c|c|c|c|c|c|}
\hline \multirow[b]{3}{*}{ Sample } & \multirow{3}{*}{$\begin{array}{l}\text { Polymer : IMC } \\
\text { weight ratio }\end{array}$} & \multirow{2}{*}{\multicolumn{2}{|c|}{ Dialysis }} & & \\
\hline & & & & \multicolumn{2}{|c|}{ Emulsion } \\
\hline & & $\mathrm{C}_{\mathrm{p}} \sim \mathrm{CAC}$ & $\mathrm{C}_{\mathrm{p}}=10 \mathrm{mg} / \mathrm{ml}$ & $\mathrm{C}_{\mathrm{p}} \sim \mathrm{CAC}$ & $\mathrm{C}_{\mathrm{p}}=10 \mathrm{mg} / \mathrm{ml}$ \\
\hline \multirow{3}{*}{ PVP-DD 25000} & $1.0: 0.0$ & $204 \pm 7$ & $412 \pm 6$ & $151 \pm 6$ & $388 \pm 3$ \\
\hline & $1.0: 0.6$ & $218 \pm 4$ & $340 \pm 5$ & $162 \pm 2$ & $302 \pm 9$ \\
\hline & $1.0: 1.0$ & $236 \pm 2$ & $248 \pm 7$ & $177 \pm 3$ & $186 \pm 6$ \\
\hline \multirow[t]{3}{*}{ PVP-DD 29000} & $1.0: 0.0$ & $237 \pm 2$ & $466 \pm 10$ & $208 \pm 5$ & $427 \pm 8$ \\
\hline & $1.0: 0.6$ & $251 \pm 2$ & $382 \pm 4$ & $229 \pm 3$ & $355 \pm 6$ \\
\hline & $1.0: 1.0$ & $270 \pm 4$ & $274 \pm 9$ & $242 \pm 8$ & $266 \pm 10$ \\
\hline \multirow[t]{3}{*}{ PVP-Hex ${ }_{2} 5000$} & $1.0: 0.0$ & $342 \pm 8$ & $587 \pm 5$ & $329 \pm 7$ & $527 \pm 10$ \\
\hline & $1.0: 0.6$ & $368 \pm 8$ & $453 \pm 7$ & $346 \pm 9$ & $409 \pm 6$ \\
\hline & $1.0: 1.0$ & N.D. & N.D. & N.D. & N.D. \\
\hline
\end{tabular}

Under these conditions the presence of hydrogen bond interactions between PVP and IMC were strong enough to prevent some IMC amount entering into the core of polymeric aggregates. When medium $\mathrm{pH}$ was increased to 7.4 the DLE and DLC increased as at these conditions it became beneficial for all the IMC to enter $\mathrm{Amph}_{2}$-PVP particles.

All obtained results of IMC-loading studies revealed that under optimal preparation conditions and polymer structure, $\mathrm{Amph}_{2}$-PVP self-assembled aggregates had stronger affinity toward the IMC, which can be successfully compared with the best results obtained previously for Amph-PVP polymers. Prepared colloidal solutions of IMC-loaded $\mathrm{Amph}_{2}$-PVP nanoparticles remained stable over four weeks at room temperature, with no material deposition and sediment observed.

Drug release study. The indomethacin release properties from $\mathrm{Amph}_{2}$-PVP and Amph-PVP nanoparticles were investigated using a dialysis membrane bags in phosphate buffer solutions ( $\mathrm{pH} 7.4,37^{\circ} \mathrm{C}$ ). Fig. 8 shows a release profile of IMC from nanoparticles with different polymer structure and DLE as a function of time. It is a plot of accumulated release as a \% of the actual IMC load, determined from the loading efficiency.

As shown in Fig. 8, while the free IMC exhibited rapid release of $98 \%$ within $24 \mathrm{~h}$, the IMC which was loaded into the inner core of $\mathrm{Amph}_{2}$-PVP and Amph-PVP showed controlled release of $45-55 \%$ for 10 days and was released slower from nano-aggregates containing higher amounts of drug. This confirmed the obtained results that increase of IMC loaded in nanoparticles enhances interaction between IMC and amphiphilic polymer, leading to decrease of drug release rate and amount.

When we compared the release behavior of $\mathrm{PVP}-\mathrm{DD}_{2}$ 5000 and PVP-OD 5000 nanoparticles, which have similar DLE and PVP fragment molecular weight, they showed also a similar release profile. From the result of this release experiment we concluded that binding affinity of IMC with din-dodecyl hydrophobic group is equal to binding affinity of IMC with n-octadecyl group and rather shorter length of each n-dodecyl group. The release profiles obtained for IMCloaded nanoparticles showed that the major factor affecting the drug release rate is binding affinity between the hydrophobic core of polymer formed by alkyl chains and drug. The molecular weight of polymeric hydrophobic fragment does not provide any significant influence on IMC release from nanoparticles.

Since the initial burst effect was very small or was not observed at all for tested polymer samples, we can conclude that $\mathrm{Amph}_{2}$-PVP nanoparticles can be prepared without any residual drug on their surface.

Thus, the release profiles observed for IMC highly-loaded nanoparticles demonstrated that $\mathrm{Amph}_{2}$-PVP nano-aggregates at certain conditions and polymer structure have enough potential to act as drug carries, as judged from their outstanding capacity to encapsulate and provide controlled release of IMC molecules.

In conclusion, amphiphilic PVP derivatives with different molecular weight of hydrophilic polymeric fragment and one end di-n-alkyl group with different length of each aliphatic chain as hydrophobic fragment were prepared using the original two-step method. Amph $\mathrm{APV}_{2}$-PVnoparticles loaded with indomethacin hydrophobic drug in their inner core were prepared by dialysis and solvent evaporation method using the solution behavior of amphiphilic polymers in selective solvents. The size of $\mathrm{Amph}_{2}$-PVP particles increases with the decrease of hydrophobic di-n-alkyl fragment length and with increasing of loading amount of drug. However, the size of IMC-loaded Amph-PVP nanoparticles can be adjusted by conditions of their preparation and polymer structure to 150-350 nm. The critical aggregation concentration values for $\mathrm{Amph}_{2}$-PVP samples are much lower than that of common low-molecular weight surfactants. The drug loading efficiency for Amph-PVP nanoparticles was up to $41.4 \%$ when drug 
loading capacity was $50 \%$. In vitro release of IMC from the IMC-loaded $\mathrm{Amph}_{2}$-PVP nanoparticles in PBS medium showed the significant sustained release behavior for most polymer samples without any burst effects. In all experiments prepared di-n-alkyl hydrophobized PVP derivatives showed good results similar to mono-n-alkyl terminated polymers and, thus, $\mathrm{Amph}_{2}$-PVP nanoparticles can be considered as promising biocompatible drug vehicles for controlled release and site-specific drug delivery systems.

\section{Acknowledgements}

This study was partially supported by the grant 'Bilateral Collaboration Greece - Russia 2004-2006' of the General Secretariat of Research and Technology, the Greek Ministry of Science and the Committee of Science and Technology of Russia.

\section{References}

1. Kwon GS and Kataoka K: Block copolymer micelles as longcirculating drug vehicles. Adv Drug Deliv Rev 16: 295-309, 1995.

2. Jones $\mathrm{M}$ and Leroux $\mathrm{J}$ : Polymeric micelles - a new generation of colloidal drug carriers. Eur J Pharm Biopharm 48: 101-111, 1999.

3. Torchilin VP: Structure and design of polymeric surfactantbased drug delivery systems. J Control Release 73: 137-172, 2001 .

4. Scholz G, Iijima M, Nagasaki Y and Kataoka K: A novel reactive polymeric micelle with aldehyde groups on its surface. Macromolecules 28: 7295-7297, 1995.

5. La SB, Okano T and Kataoka K: Preparation and characterization of the micelle-forming polymeric drug indomethacinincorporated poly(ethylene oxide)-poly(ß-benzyl-L-aspartate) block copolymer micelles. J Pharm Sci 85: 85-90, 1996.

6. Gregoriadis G: Targeting of drugs. Nature 265: 407-411, 1977.

7. Weissig V, Lizano C and Torchilin VP: Micellar delivery system for dequalinium - a liphophilic cationic drug with anticarcinoma activity. J Liposome Res 8: 391-400, 1998.

8. Kwon G, Suwa S, Yokohama M, Okano T, Sakurai Y and Kataoka K: Enhanced tumor accumulation and prolonged circulation times of micelle-forming poly(ethylene oxideaspartate) block copolymer-adriamycin conjugates. J Control Release 29: 17-23, 1994.

9. Yokohama M, Miyauchi M, Yamada N, Okano T, Sakurai Y, Kataoka $\mathrm{K}$ and Inoue $\mathrm{S}$ : Characterization and anticancer activity of the micelle-forming polymeric anticancer drug adriamycinconjugated poly(ethylene glycol)-poly(aspartic acid) block copolymer. Cancer Res 50: 1693-1700, 1990.

10. Dowling KC and Thomas JK: A novel micellar synthesis and photophysical characterization of water-soluble acrylamidestyrene block copolymers. Macromolecules 23: 1059-1064, 1990.

11. Honda C, Hasegawa Y, Hirunuma R and Nose T: Micellization kinetics of block copolymers in selective solvent. Macromolecules 27: 7660-7668, 1994.

12. Jones JL, Marques CM and Joanny JF: Shear-induced micellization of diblock copolymers. Macromolecules 28: 136-142, 1995.

13. Park TG: Degradation of poly(lactic-co-glycolic acid) microspheres: effect of copolymer composition. Biomaterials 16: 1123-1130, 1995.

14. Huatan H, Collett JH and Attwood D: The microencapsulation of protein using a novel ternary blend based on poly( $\varepsilon$-caprolactone). J Microencapsul 12: 557-567, 1995.
15. Lin WJ, Donglas R and Linhardt RT: Accelerated degradation of poly ( $\varepsilon$-caprolactone) by organic amines. Pharm Res 11: 1030-1035, 1994.

16. Bergsma JE, Rozema FR, Bos RRM, Boering G, Joziasse CAP and Pennings AJ: In vitro predegradation at elevated temperatures of poly (lactide). J Mater Sci Mater Med 6: 642-646, 1995.

17. Trubetskoy VS and Torchilin VP: Polyethyleneglycol based micelles as carriers of therapeutic and diagnostic agents. STP Pharma Sci 6: 79-86, 1996.

18. Verdun C, Couvreur P, Vrancks H, Lenaerts V and Roland M: Development of a nanoparticle controlled release formulation for human use. J Control Release 3: 205-210, 1986.

19. Holland SJ, Tighe BJ and Gould PL: Polymers for biodegradable medical devices. 1. The potential of polyesters as controlled macromolecular release systems. J Control Release 4: 155-180, 1986.

20. Leroux JC, Allemann E, Taeghere FD, Doelker E and Gurny R: Biodegradable nanoparticles from sustained release formations to improved site specific drug delivery. J Control Release 39: 339-350, 1996.

21. Dreschler H: Indication to prescription of blood and blood substitutes in operative medicine. Med Lab (Stuttg) 27: 115-121, 1974.

22. Kramer SA: Effect of povidone-iodine on wound healing: a review. J Vasc Nurs 17: 17-23, 1999.

23. Kuskov AN, Shtilman MI, Goryachaya AV, Tashmuhamedov RI, Yaroslavov AA, Torchilin VP, Tsatsakis AM and Rizos AK: Self-assembling nano-scaled drug delivery systems composed of amphiphilic poly-N-vinylpyrrolidones. J Non-Cryst Solids 353: 3969-3975, 2007.

24. Fawaz F, Bonini F, Guyot M and Lagueny AM: Influence of poly(DL-lactide) nanocapsules on the biliary clearance and enterohepatic circulation of indomethacin in the rabbit. Pharm Res 10: 750-756, 1993.

25. Kuskov AN, Villemson AL, Shtilman MI, Larionova NI, Tsatsakis AM, Tsikalas I and Rizos AK: Amphiphilic poly-Nvinylpyrrolidone nano-carriers with incorporated model proteins. J Phys Condens Matter 19: 5139-5150, 2007.

26. Roy S, Pal M and Gupta BK: Indomethacin-loaded microspheres: Design and preparation by a multiple-emulsification technique and their in vitro evaluation. Pharm Res 9: 1132-1136, 1992.

27. Shin I, Kim S, Lee Y, Cho C and Sung Y: Methoxy poly(ethylene glycol $) / \varepsilon$-caprolactone amphiphilic block copolymeric micelle containing indomethacin: I. preparation and characterization. J Control Release 51: 1-11, 1998.

28. Kim S, Shin I, Lee Y, Cho C and Sung Y: Methoxy poly(ethylene glycol)/ $\varepsilon$-caprolactone amphiphilic block copolymeric micelle containing indomethacin: II. Micelle formation and drug release behaviours. J Control Release 51: 13-22, 1998.

29. Kuskov AN, Voskresenskaya AA, Goryachaya AV, Artyukhov AA, Shtilman MI and Tsatsakis AM: Preparation and characterization of amphiphilic poly-N-vinylpyrrolidone nanoparticles containing indomethacin. J Mater Sci Mater Med DOI 10.1007/s10856-010-4029-1, 2010.

30. Yekta A, Duhamel J, Brochard P, Adiwidjajja H and Winnik MA: A fluorescent probe study of micelle-like cluster formation in aqueous solutions of hydrophobically modified poly(ethylene oxide). Macromolecules 26: 1829-1836, 1993.

31. Astafieva I, Zhong XF and Eisenberg A: Critical micellization phenomena in block polyelectrolyte solutions. Macromolecules 26: 7339-7352, 1993.

32. Kim S, Shin I and Lee Y: Preparation and characterization of biodegradable nanospheres composed of methoxy poly(ethylene glycol) and D,L-lactide block copolymer as novel drug carriers. J Control Release 56: 197-208, 1998.

33. Giacomelli C, Schmidt V and Borsali R: Specific interactions improve the loading capacity of block copolymer micelles in aqueous media. Langmuir 23: 6947-6955, 2007.

34. Yang L, Kuang J, Wang J, Li Z and Zhang L: Loading and in vitro controlled release of indomethacin using amphiphilic cholesteryl-bearing carboxymethylcellulose derivatives. Macromol Biosci 8: 279-286, 2008. 